\title{
Exposure rate to zika virus infection in a sample of clinically diagnosed dengue patients presenting to the Teaching Hospital, Jaffna, Sri Lanka
}

\author{
K Murugananthan ${ }^{1}$, R Kowsalavani $^{2}$
}

\section{Introduction}

Zika virus (ZV) and dengue virus (DENV) infections are arthropod borne infections transmitted by the Aedes mosquitoes. These infections produce similar clinical manifestations during the early phase of the illness which could possibly result in misdiagnosis of zika virus infection as dengue virus infection. We therefore undertook this study to identify previous exposure to zika virus infection among patients with suspected dengue in Jaffna, Sri Lanka.

\section{Methods}

Ninety one serum samples from clinically suspected dengue patients were subjected to ELISA for the detection of anti-ZV IgM and anti-ZV IgG. The evidence of past exposure to ZV was determined through anti-ZV IgG positivity. Anti-ZV IgM was considered as evidence for more recent ZV infections. Correlation of clinical/demographic features with anti-ZV IgM were determined. The presence of DENV and ZV co-infection was detected using anti-DENV IgM (previously available data) and anti-ZV IgM positivity in the study group. The past exposure rates were determined through anti-DENV IgG (previously available data) and anti-ZV virus $\operatorname{IgG}$ positivity.

\section{Results}

Of the 91 patients, $1.1 \%$ had evidence for acute $\mathrm{ZV}$ infection and $19.8 \%$ had evidence for past ZV infection. The overall exposure rate to $\mathrm{ZV}$ infection (anti-ZV IgM and IgG positivity) in the population was $20.9 \%$. Though the whole study group was clinically diagnosed as dengue, only 69.2\% had acute DENV infection. None of the patients had DENV and ZV co-infection based on anti-DENV IgM and anti-ZV IgM positivity. Based on anti-DENV IgG and anti-ZV IgG, 13.2\% had evidence of previous exposure. The patient detected with acute $\mathrm{ZV}$ infection presented with 11 days of fever, myalgia, dyspnoea and flushed skin.

\section{Conclusions}

Findings of this study show evidence for the presence of DENV and ZV infection. One fifth of the study population had more recent or past ZV infection. However, we cannot say whether they were co-infected (concurrently infection) or infected with the two viruses at different times. Laboratory diagnosis of $\mathrm{ZV}$ infection must be done in dengue suspected patients in the study area.

Keywords: Zika virus, Dengue virus, Co-infection

\footnotetext{
${ }^{1}$ Department of Microbiology, Faculty of Medicine, University of Jaffna, Sri Lanka

${ }^{2}$ Department of Biochemistry and Molecular Biology, Postgraduate Institute of Science, University of Peradeniya, Sri Lanka

Address for correspondence: Dr.K Murugananthan.Telephone:+94718742349 Email: kalamathy6@gmail.com (iD https://orcid.org/https://orcid.org/0000-0003-2223-9728
} 